\title{
Group Selection and Reciprocity among Kin
}

\author{
Neelesh Dahanukar ${ }^{*}, 1,2$ and Milind Watve ${ }^{1,2}$ \\ ${ }^{1}$ Indian Institute of Science Education and Research, Sai Trinity, Central Tower, Garware Circle, Pune 411021, India \\ ${ }^{2}$ Department of Microbiology, Abasaheb Garware College, Karve Road, Pune 411004, India
}

\begin{abstract}
The question how Darwinian mechanisms lead to the evolution of individually costly cooperative behavior has given rise to a number of hypotheses. However, attempts to build a synthesis where different types of mechanisms coexist and interact at different levels of selections are still scarce. Here we derive simple game theoretical models where the group level conflicts are resolved by group selection while simultaneously within group competition is resolved by kin selection and reciprocity. We show that none of the mechanisms, when alone, is as robust in evolving and maintaining cooperation as a synthesis of all. Furthermore, we show that initially within group conflicts can be overcome only by kin selection and not reciprocity. However, once common, different types of reciprocities can maintain high levels of cooperation even if average relatedness among individuals is lowered, groups become large, and the benefits of cooperation are reduced. Based on the synthesis we also propose a possible route to the evolution of social and eusocial systems.
\end{abstract}

Keywords: Evolutionary game theory, prisoner's dilemma, evolution of cooperation, multi-level selection, evolution of social systems.

\section{INTRODUCTION}

Evolution of cooperation in the Darwinian struggle for existence is an enduring evolutionary conundrum and a number of solutions are proposed. While the numbers of possible solutions are overwhelming, we can identify two schools of thoughts where the difference lies in whether a group or an individual is the unit of selection. Group selection theory predicts that group beneficial traits will evolve due to competition between groups [1]. However, it falls short when explaining how such traits will be maintained when within group individual level selection does not favor their evolution $[2,3]$. Several theories have been proposed to resolve this problem of individual level selection, of which two of the most celebrated theories are kin selection and reciprocity. If the cost to benefit ratio is smaller than the average relatedness between individuals then kin selection can favor evolution of cooperation [4], while, among the unrelated individuals, cooperation can evolve through reciprocity [5] where individuals cooperate with those who have cooperated with them before (direct reciprocity) [6], or cooperate with reputed cooperators (indirect reciprocity) [7] or evolve cooperation by punishing the defectors (strong reciprocity) $[8,9]$.

Kin selection theory has been the subject of heated debate in the past few years with studies both supporting and criticizing its applicability in the evolution of cooperation. It is suggested that the tradeoff between direct and indirect gains and competition between kin can disrupt kin-based cooperation [10]. A major debate was started by Wilson [11] and Wilson and Hölldobler, [12] who said that kin selection

*Address correspondence to this author at the Indian Institute of Science Education and Research, Sai Trinity, Central Tower, Garware Circle, Pune 411021, India; E-mail: neeleshdahanukar@rediffmail.com is not a key to altruism in social insects. Rather, group selection could have been the driving force behind evolution of cooperation, so that genetic relatedness is just a consequence of the phenomenon. Foster et al. [13] strongly criticized this claim. Flecher et al. [14] pleaded for the importance of reciprocity over kin selection, which was again counter attacked by Foster et al. [15], leaving the debate far from resolved. There has also been an attempt to show that both kin selection and group selection work on the same principle [16].

We believe that while all the suggestions are valid, favoring one theory over another may not give us a clue on the evolution of cooperation in natural environments. In nature it is likely that several different mechanisms work at the same time to bolster cooperative organizations. What we need, therefore, is a synthesis in which several mechanisms of evolution of cooperation coexist at the same time. There have been some attempts in this respect. Lehmann and Keller [17] give a general framework for the evolution of cooperation by incorporating several mechanisms in a single equation and show how the different models of cooperation are special cases of this equation. Meanwhile Marshall and Rowe [18] and Fletcher and Zwick [19] synthesized models that incorporate both kin selection and direct reciprocity to study the interactions between the two mechanisms and understand the basic principles underlying these mechanisms respectively. Even though these models attempt to unify the concepts and understand how different mechanisms interact with each other, they have given little attention to two important behaviors: namely punishment and discrimination (individual recognition and memory over time by others in the interacting group). Our study differs from other studies in three respects. Firstly, we build simple game theoretical matrix models by incorporating group selection, kin selection and different types of reciprocity and study the 
conditions that favor evolution of cooperation in a population of defectors and the conditions that maintain cooperation when it is common. Secondly, based on our findings we propose a possible route to the co-evolution of strategies for cooperation and social systems. Third, by integrating dyadic interaction with group behavior, we show the role of individual recognition and social signaling, making it possible to recruit allies to punish defectors either by other members of an individual's kin, or by collective behaviors of a group (of which ostracism plays a hitherto largely unnoted role) [20].

In this study we derive simple game theoretical models incorporating kin selection, group selection and different types of reciprocities. We show that group selection with individual level conflict resolved by kin selection evolves more robust cooperative organization than either of the mechanisms alone. Further, we show that reciprocity cannot evolve cooperation in a population of defectors; but if the degree of relatedness is more than the cost to benefit ratio, kin selection can reinforce cooperation which-once it's common-can be maintained by direct, indirect, or strong reciprocity even when average relatedness is lowered. Thus group selection and reciprocity among kin is robust to variability in gains from cooperative act. Based on our findings we propose possible route to the evolution of social and eusocial systems. We also substantiate our proposals using empirical evidence.

\section{Prisoner's Dilemma}

Prisoner's dilemma is used as a major paradigm to study the evolution of cooperation. In a two person Prisoner's Dilemma (PD) game both players have two behavioral options, either to cooperate (C) or to defect (D). Let ' $b$ ' be the benefit of a cooperative act and ' $c$ ' be the cost of cooperation such that $b>c>0$. In a PD interaction the benefit of a cooperative act is only to the opponent while the cooperator pays the cost of cooperation. Thus, if both the players cooperate mutually then both achieve the payoff $b-c$, while if both defect both get nothing. If one player cooperates while the other defects then the cooperator gets $-c$ and the defector gets $b$. This can be put in a matrix form as follows.

\begin{tabular}{c|cc} 
& $C$ & $D$ \\
\hline$C$ & $b-c$ & $-c$ \\
$D$ & $b$ & 0
\end{tabular}

As defection always pays better, irrespective of the behavioral choice of the opponent, defection is commonly viewed as the only evolutionary stable Nash Equilibrium in this game. While this is true for two isolated individuals in a single interaction, repeated interactions and the presence of an observer who can identify and remember the defector and exclude those engaging in defection (a frequent characteristic in animal groups and ubiquitous among humans due to the evolution of language and naming of both individuals and kin groups) can convert the standard PD into a cooperative game. As a result, models of cooperation differ greatly once individual behavioral responses are integrated with the likely behaviors of members of a group in species with individual recognition and memory (as is found in many primates and in humans). We will incorporate these complexities in the basic model in a stepwise manner.

\section{Kin Selection in Prisoner's Dilemma}

Starting with the standard model of an interaction between two isolated individuals without an observer, we model kin selection in the PD game as follows. If the average relatedness between individuals is $r$ then the gains and losses of the opponent are shared by the focal individual with the fraction $r$ [21-23]. The resultant payoff matrix is

$$
\begin{array}{c|cc} 
& C & D \\
\hline C & (b-c)(1+r) & r b-c \\
D & b-r c & 0
\end{array}
$$

We call this model as the 'relatedness coefficient model' where we consider a linear weighting approach. Even though we have used this approach in the next six sections we will also consider another model where we will treat $r$ as a positive assortment probability leading to a non-linear weighting approach.

We will analyze the model for stability of cooperation and invasion of defection. If $\mathrm{E}(\mathrm{X} \mid \mathrm{Y})$ is the payoff of a person adopting strategy $\mathrm{X}$ when in a population using strategy $\mathrm{Y}$, then cooperation will be ESS (evolutionarily stable strategy) [24], if $\mathrm{E}(\mathrm{C} \mid \mathrm{C})>\mathrm{E}(\mathrm{D} \mid \mathrm{C})$ while cooperators can invade a population of defectors if $\mathrm{E}(\mathrm{C} \mid \mathrm{D})>\mathrm{E}(\mathrm{D} \mid \mathrm{D})$. In the presence of kin selection cooperation will be stable and can invade a population of defectors in the PD game if $r>c / b$ (Table 1).

\section{Group Selection among Kin}

One extension of group selection model has been presented by Nowak [23], who reduces the evolutionary dynamics of individual level selection within the group and group level selection between groups to a single two-person game on one level of selection. Consider a population which is subdivided into $m$ groups. The maximum size of a group is $n$. Individuals in the same group interact with each other through Prisoner's Dilemma game given by the payoff matrix (1). We begin with the standard "one-shot" twoperson PD without an observer, whose actors lack all memory that will influence behavior in any possible future interaction that is mutually beneficial. At each time step, an individual from the entire population is chosen for reproduction proportional to its inclusive fitness (i.e., potential to increase the transmission of "genes identical by descent"-and hence including the survival and reproduction of close kin). In this two-person model, offspring are added to the same group (i.e., all mating is endogamous).

Between groups there is no game dynamical interaction, but groups divide at rates that are proportional to the average fitness of individuals in that group. (Note this means the model excludes exogamous mating between local bands or members of different clans in a pre-literate tribe or segmentary lineage system; once these complexities of human kinship made possible by language and memory, as we will show, it is necessary to integrate the levels of analysis as we propose). In the model restricted to single, entirely endogamous groups, one can say that cooperator groups have a constant payoff $b$-c, while defector groups 
Table 1. Payoff Matrices and Conditions for Maintenance of Cooperation and Invasion for Defectors in Prisoner's Dilemma Played between Kin

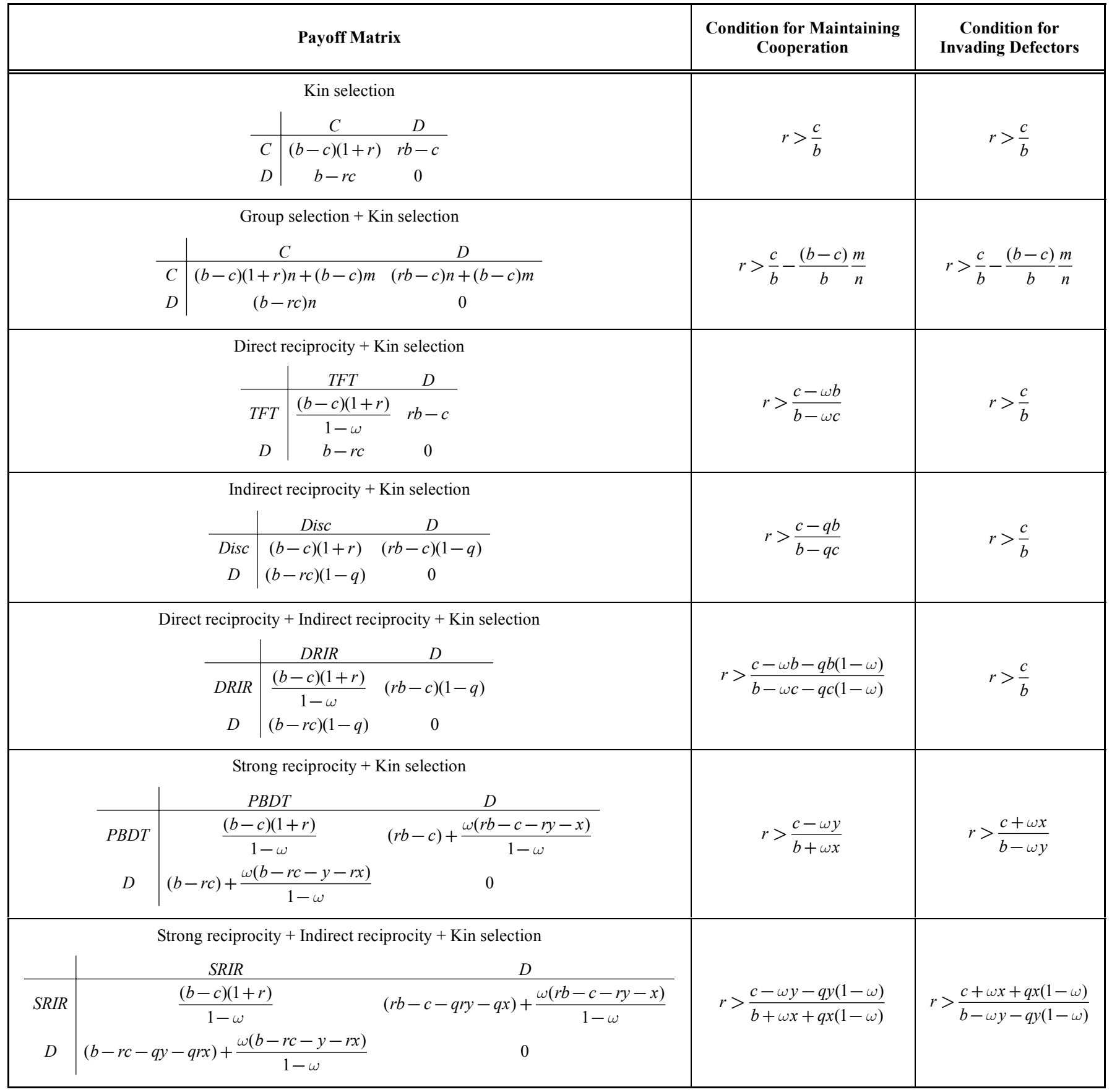

have a constant payoff 0 . Hence, in a sense between groups the game can take the form as follows,

$$
\begin{array}{c|cc} 
& C & D \\
\hline C & (b-c) & (b-c) \\
D & 0 & 0
\end{array}
$$

We can now multiply the matrix (1) by the group size, $n$, and the matrix (3) by the number of groups, $m$, and add the two matrices. The result is,

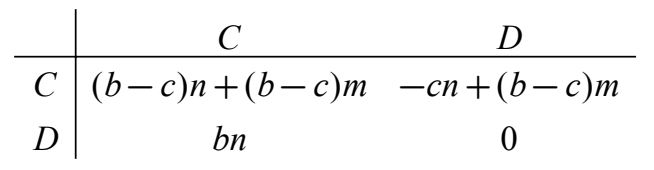

Cooperation will be stable if $\mathrm{E}(\mathrm{C} \mid \mathrm{C})>\mathrm{E}(\mathrm{D} \mid \mathrm{C})$ and cooperators will invade defectors if $\mathrm{E}(\mathrm{C} \mid \mathrm{D})>\mathrm{E}(\mathrm{D} \mid \mathrm{D})$. Both these conditions are satisfied when $m / n>c /(b-c)$ [23].

We will now consider that genetically related individuals coalesce in the same group and interact with each other through Prisoner's Dilemma game given by the payoff 
matrix (2). If the average relatedness between individuals of two different groups is negligibly small (as occurs without exogamy) then the payoff matrix (3) that describes the payoff between groups will not change. Now, we can multiply matrix (2) by the group size, $n$, and the matrix (3) by the number of groups, $m$, and add the two matrices. The result is,

\begin{tabular}{c|cc} 
& $C$ & $D$ \\
\hline$C$ & $(b-c)(1+r) n+(b-c) m$ & $(r b-c) n+(b-c) m$ \\
$D$ & $(b-r c) n$ & 0
\end{tabular}

Cooperation will be stable if $\mathrm{E}(\mathrm{C} \mid \mathrm{C})>\mathrm{E}(\mathrm{D} \mid \mathrm{C})$ and cooperators will invade defectors if $\mathrm{E}(\mathrm{C} \mid \mathrm{D})>\mathrm{E}(\mathrm{D} \mid \mathrm{D})$. Both these conditions are satisfied when $m / n>(c-r b) /(b-c)$. In terms of $r$ the equation can be rearranged as $r>\frac{c}{b}-\frac{(b-c)}{b} \frac{m}{n}$.

In the absence of kin selection, group selection can evolve cooperation and maintain it if $\frac{m}{n}>\frac{c}{b-c}$ [23]. In the presence of kin selection, the condition for the evolution and maintenance of cooperation is $\frac{m}{n}>\frac{c-r b}{b-c}$. The equation depicts that as the relatedness between individuals increases the threshold value of $m / n$ will decrease, indicating that kin selection can evolve and maintain cooperation within groups when groups are large and the number of groups is small. Thus, group selection works better with kin selection than alone for interacting individuals with any $r>0$. Furthermore, the threshold value of relatedness required in the presence of both kin selection and group selection is $r>\frac{c}{b}-\frac{(b-c)}{b} \frac{m}{n}$.

Thus, kin selection works well in the presence of group selection than alone for any $b>c$. Hence, group and kin selection together can evolve robust cooperation than either of them working alone, especially when average relatedness is low, groups are large and the number of groups is small.

A plot of threshold value of relatedness required to evolve and maintain cooperation with variable $b, n$ and $m$ values is shown in Fig. (1), which depicts that small group sizes favor cooperation at low relatedness even when benefits from cooperative act are small. This explains why nonhuman primates and preliterate human tribes normally form small hunter-gatherer bands. When the group size increases, cooperation is favored only if the number of competing groups is large (as occurs among mammals forming herds whose size creates limitations to the total population sustainable in a specific location).

While we begin with this model, showing how it leads to the need to combine group selection and the individual's cost-benefit calculus, we then add the implications of an observer who can communicate the outcome of a single PD interaction to other members of the group with whom the
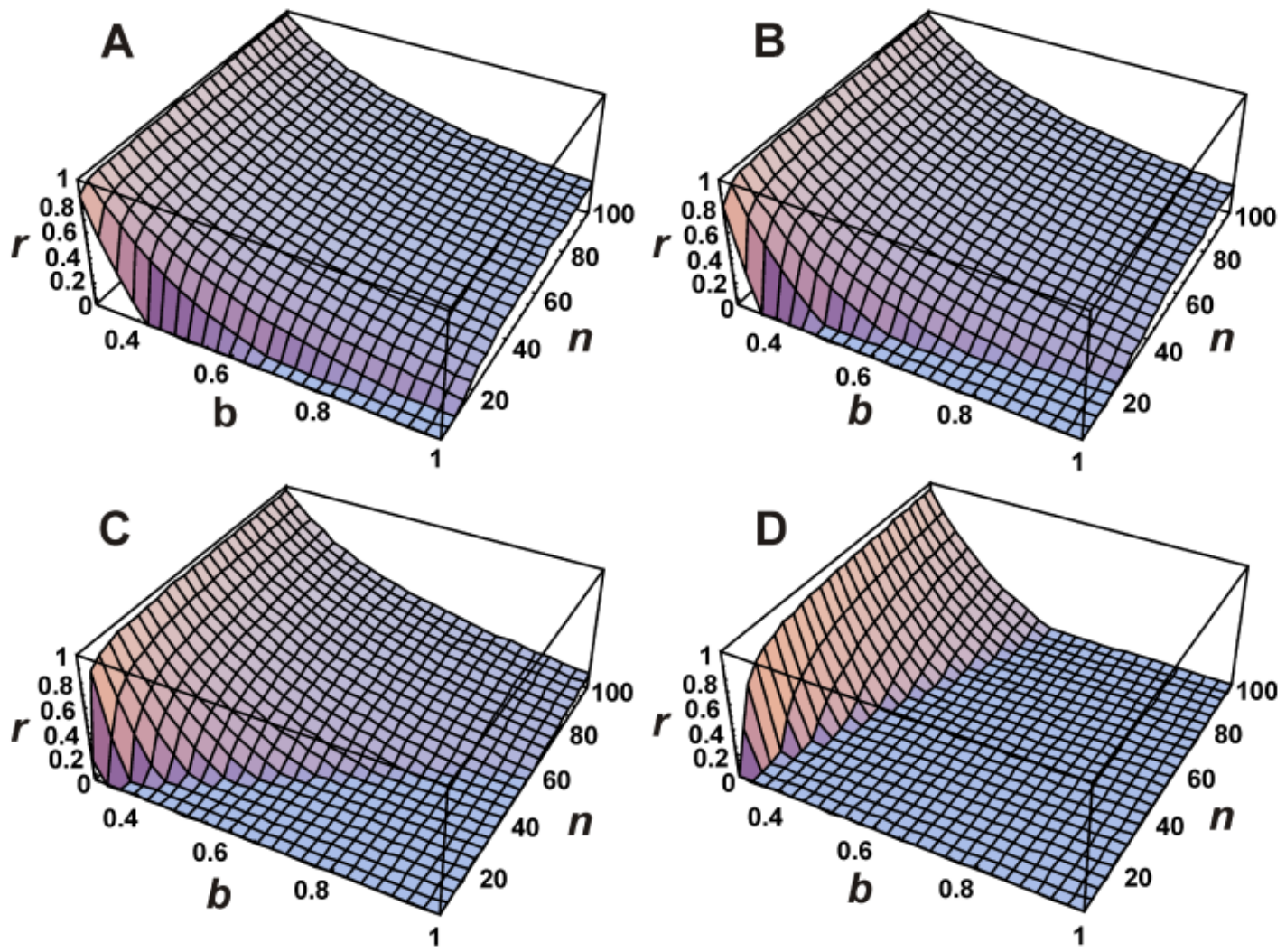

Fig. (1). Effect of group size $(n)$ and benefit of cooperation $(b)$ on the threshold relatedness $(r)$ required to evolve cooperation for different number of groups $(m)$ when cooperators use both direct and indirect reciprocity. (A) $m=5,(\mathbf{B}) m=10,(\mathbf{C}) m=25$ and $(\mathbf{D}) m=100$. Cost of cooperation $(c)$ is 0.3 . 
defector will necessarily interact in the future. In this new model, combining individual recognition with memory shows how the kinship between the cooperator and the observer combined with prediction of future social interaction transforms even a single-shot PD.

\section{Direct Reciprocity among Kin}

At the individual level, we will now consider that individuals use direct reciprocity with their kin. One of the simplest strategies of direct reciprocity is Tit-For-Tat (TFT) where the player cooperates in the first move and then onwards repeats the same strategy as the opponent's previous move [6]. We will consider that all the cooperators are using TFT strategy. Let $\omega^{\mathrm{n}-1}$ be the probability that $\mathrm{n}^{\text {th }}$ interaction with the same opponent really occurs (a probability greatly increased by the enhanced memory and cognition found in great apes and especially humans). Two TFT players will cooperate in all the interactions so,

$$
\begin{aligned}
& E(T F T \mid T F T)=(b-c)+\omega(b-c) \\
& \quad+\omega^{2}(b-c)+\omega^{3}(b-c)+\ldots .=\frac{b-c}{1-\omega}
\end{aligned}
$$

However, when in contest with the D player, TFT player will cooperate in the first interaction but will defect in the subsequent interactions, so

$$
E(T F T \mid D)=(-c)+\omega(0)+\omega^{2}(0)+\omega^{3}(0)+\ldots . .=-c
$$

While, the defector will get,

$$
E(D \mid T F T)=(b)+\omega(0)+\omega^{2}(0)+\omega^{3}(0)+\ldots . .=b
$$

Two D players will always defect, so

$$
E(D \mid D)=(0)+\omega(0)+\omega^{2}(0)+\omega^{3}(0)+\ldots . .=0
$$

Thus the payoff matrix between a cooperator using TFT strategy and a defector will be given as,

\begin{tabular}{c|cc} 
& $T F T$ & $D$ \\
\hline$T F T$ & $\frac{(b-c)}{1-\omega}$ & $-c$ \\
$D$ & $b$ & 0
\end{tabular}

TFT will be stable against defectors when E (TFT $\mid$ TFT $)>$ $\mathrm{E}(\mathrm{D} \mid \mathrm{TFT})$, i.e. when $\omega>c / b$. However, TFT players cannot invade a population of defectors as $E(D \mid D)>E$ (TFT|D) for any $\mathrm{c}>0$. If mechanisms of individual recognition, identification of kin, and memory are present (as in humans), this means that TFT is likely to generate group selection due to differential signaling for members of a local group as compared to outsiders. (Evolved traits, such as suspicion of outsiders whose phenotypical traits are unfamiliar, probably reflect this linkage between TFT and group selection). then,

Now consider that the interactions are among relatives

$$
\begin{aligned}
E(T F T \mid T F T)= & (b-c)(1+r)+\omega(b-c)(1+r) \\
& +\omega^{2}(b-c)(1+r)+\ldots .=\frac{(b-c)(1+r)}{1-\omega}
\end{aligned}
$$

$$
\begin{gathered}
E(T F T \mid D)=(r b-c)+\omega(0)+\omega^{2}(0) \\
+\omega^{3}(0)+\ldots . .=r b-c \\
\begin{aligned}
E(D \mid T F T)=(b-r c)+\omega(0)+\omega^{2}(0) \\
+\omega^{3}(0)+\ldots . .=b-r c
\end{aligned} \\
E(D \mid D)=(0)+\omega(0)+\omega^{2}(0)+\omega^{3}(0)+\ldots . .=0
\end{gathered}
$$

Thus the payoff matrix takes the form,

\begin{tabular}{c|cc} 
& $T F T$ & $D$ \\
\hline$T F T$ & $\frac{(b-c)(1+r)}{1-\omega}$ & $r b-c$ \\
$D$ & $b-r c$ & 0
\end{tabular}

Here, TFT will be stable against defectors when $\mathrm{E}$ $(\mathrm{TFT} \mid \mathrm{TFT})>\mathrm{E}(\mathrm{D} \mid \mathrm{TFT})$, i.e. when $r>\frac{c-\omega b}{b-\omega c}$ and TFT players will invade a population of defectors when $\mathrm{E}$ $(\mathrm{TFT} \mid \mathrm{D})>\mathrm{E}(\mathrm{D} \mid \mathrm{D})$ ie., when $r>c / b$. Thus, as the probability that the two players meet again increases, cooperation can be maintained even when the relatedness among individuals is low. Once again, this prediction will be strengthened by reliable identification and memory of both kinship and group membership.

The threshold value of relatedness above which cooperation is stable for different values of benefit of cooperation (b) and probability of meeting again $(\omega)$ is given in Fig. (2).

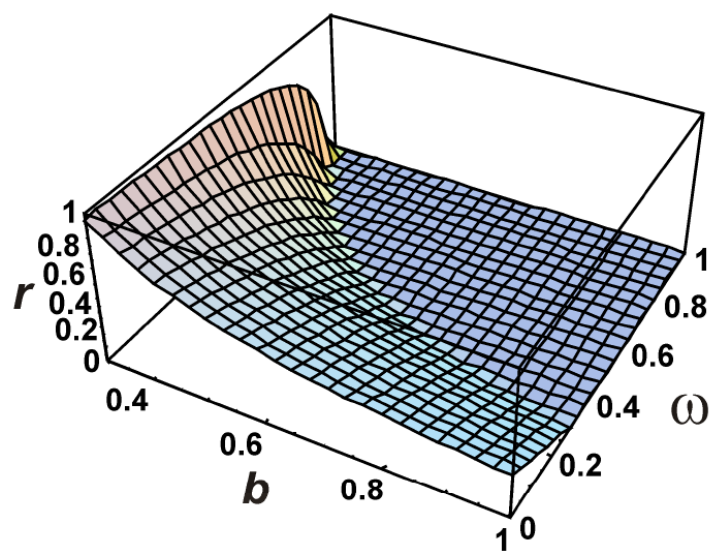

Fig. (2). Effect of probability of meeting the same person again $(\omega)$ and benefit of cooperation $(b)$ on the threshold relatedness $(r)$ required for maintaining cooperation when cooperators use TFT strategy. Cost of cooperation (c) is 0.3 . 
The figure depicts that when probability of meeting the same opponent is high, cooperation can evolve even at low relatedness and small benefit of cooperation. However, TFT will invade the population of defectors only if $r>c / b$, indicating that cooperators can invade defectors only through kin selection and direct reciprocity by itself cannot evolve cooperation.

\section{Indirect Reciprocity among Kin}

Indirect reciprocity is a form of reciprocity where cooperators build reputation and discriminators in the population cooperate only with the reputed cooperators [7]. This type of reciprocity does not depend upon the probability of meeting the same player again, but it works only if there are discriminators who can discriminate between the cooperators and defectors. We will consider indirect reciprocity model similar to Nowak and Sigmund [7] where cooperation increases ones own reputation, while defection reduces it. If there are discriminating individuals in the population who discriminate between individuals having good reputation and bad reputation and cooperate only with the players having good reputation, then cooperation can evolve through indirect reciprocity. We will consider that all the cooperators exist as discriminators (Disc). Let $q$ be the probability that a discriminator can discriminate between good and bad reputed individuals $(0 \leq q \leq 1)$, then the payoff matrix between discriminators and defectors can be derived as follows. Two discriminators will always cooperate with each other and with a defector with a probability 1-q. Thus,

\begin{tabular}{c|cc} 
& Disc & $D$ \\
\hline Disc & $(b-c)$ & $-c(1-q)$ \\
$D$ & $b(1-q)$ & 0
\end{tabular}

Here, cooperation will be stable if $\mathrm{E}$ (Disc $\mid$ Disc) $>\mathrm{E}$ (D $\mid$ Disc), ie. when $q>c / b$. However, cooperators cannot invade defectors because $\mathrm{E}(\mathrm{D} \mid \mathrm{D})>\mathrm{E}(\operatorname{Disc} \mid \mathrm{D})$ for any $\mathrm{c}>0$ and $\mathrm{q}<1$.

Now let us consider indirect reciprocity between relatives. A discriminator will cooperate with the defector with probability $1-q$ and the gains and losses of both the individuals will be shared with each other proportional to the average relatedness between them. Thus,

\begin{tabular}{c|cc} 
& Disc & $D$ \\
\hline Disc & $(b-c)(1+r)$ & $(r b-c)(1-q)$ \\
$D$ & $(b-r c)(1-q)$ & 0
\end{tabular}

Cooperation based on discrimination strategy will be stable if $\mathrm{E}(\operatorname{Disc} \mid \operatorname{Disc})>\mathrm{E}(\mathrm{D} \mid \mathrm{Disc})$, ie. when $r>\frac{c-q b}{b-q c}$, while cooperators will invade a population of defectors if $E$ $(\operatorname{Disc} \mid \mathrm{D})>\mathrm{E}(\mathrm{D} \mid \mathrm{D})$, ie. when $r>c / b$. These conditions are similar to the conditions achieved for direct reciprocity where $q$ is replaced by $\omega$. Thus, if the probability of discrimination is more, cooperation can be maintained through indirect reciprocity even when relatedness and gains from cooperation are low. However, discrimination itself cannot evolve cooperation and cooperation can evolve only through kin selection.

\section{Direct and Indirect Reciprocity among Kin}

A major drawback of direct reciprocity by TFT strategy is that in the absence of human linguistic and cultural symbols of kinship and memory discussed above, it always cooperates with the opponent in the first round. This could be a major loss if the opponent is a defector. In such a condition, knowing the strategy of the opponent by discrimination can always be beneficial. If a TFT player can discriminate between the cooperator and a defector, it will avoid being exploited even in the first move. We can thus merge direct and indirect reciprocity and come up with a strategy DRIR which will discriminate and cooperate with only cooperators. Similar to the derivation of payoff matrix for direct reciprocity and indirect reciprocity among kin we can derive the payoff matrix for DRIR strategy among kin as,

$$
\begin{gathered}
E(D R I R \mid D R I R)=(b-c)(1+r)+\omega(b-c)(1+r) \\
+\omega^{2}(b-c)(1+r)+\ldots . .=\frac{(b-c)(1+r)}{1-\omega} \\
\begin{array}{r}
E(D R I R \mid D)=(r b-c)(1-q)+\omega(0)+\omega^{2}(0) \\
+\omega^{3}(0)+\ldots . .
\end{array}=(r b-c)(1-q) \\
E(D \mid D R I R)=(b-r c)(1-q)+\omega(0)+\omega^{2}(0) \\
+\omega^{3}(0)+\ldots . .=(b-r c)(1-q) \\
E(D \mid D)=(0)+\omega(0)+\omega^{2}(0)+\omega^{3}(0)+\ldots . .=0
\end{gathered}
$$

In the matrix form,

\begin{tabular}{c|cc} 
& DRIR & $D$ \\
\hline$D R I R$ & $\frac{(b-c)(1+r)}{1-\omega}$ & $(r b-c)(1-q)$ \\
$D$ & $(b-r c)(1-q)$ & 0
\end{tabular}

Here, DRIR will maintain cooperation in the population if $\mathrm{E}(\mathrm{DRIR} \mid \mathrm{DRIR})>\mathrm{E}$ (D|DRIR), i.e. when $r>\frac{c-\omega b-q b(1-\omega)}{b-\omega c-q c(1-\omega)}$, indicating that both $\omega$ and $q$ can decrease the threshold relatedness for any $b>c$.

Effect of $\omega, q$ and $b$ on the threshold value of $r$ required to evolve cooperation using DRIR strategy is shown in Fig. (3). The figure depicts that direct and indirect reciprocity among kin can be a very robust means of maintaining cooperation even when the benefit of cooperation is small. However, it will invade the population of defectors only if $\mathrm{E}$ $(\mathrm{DRIR} \mid \mathrm{D})>\mathrm{E}(\mathrm{D} \mid \mathrm{D})$, i.e. when $r>c / b$ suggesting that only 

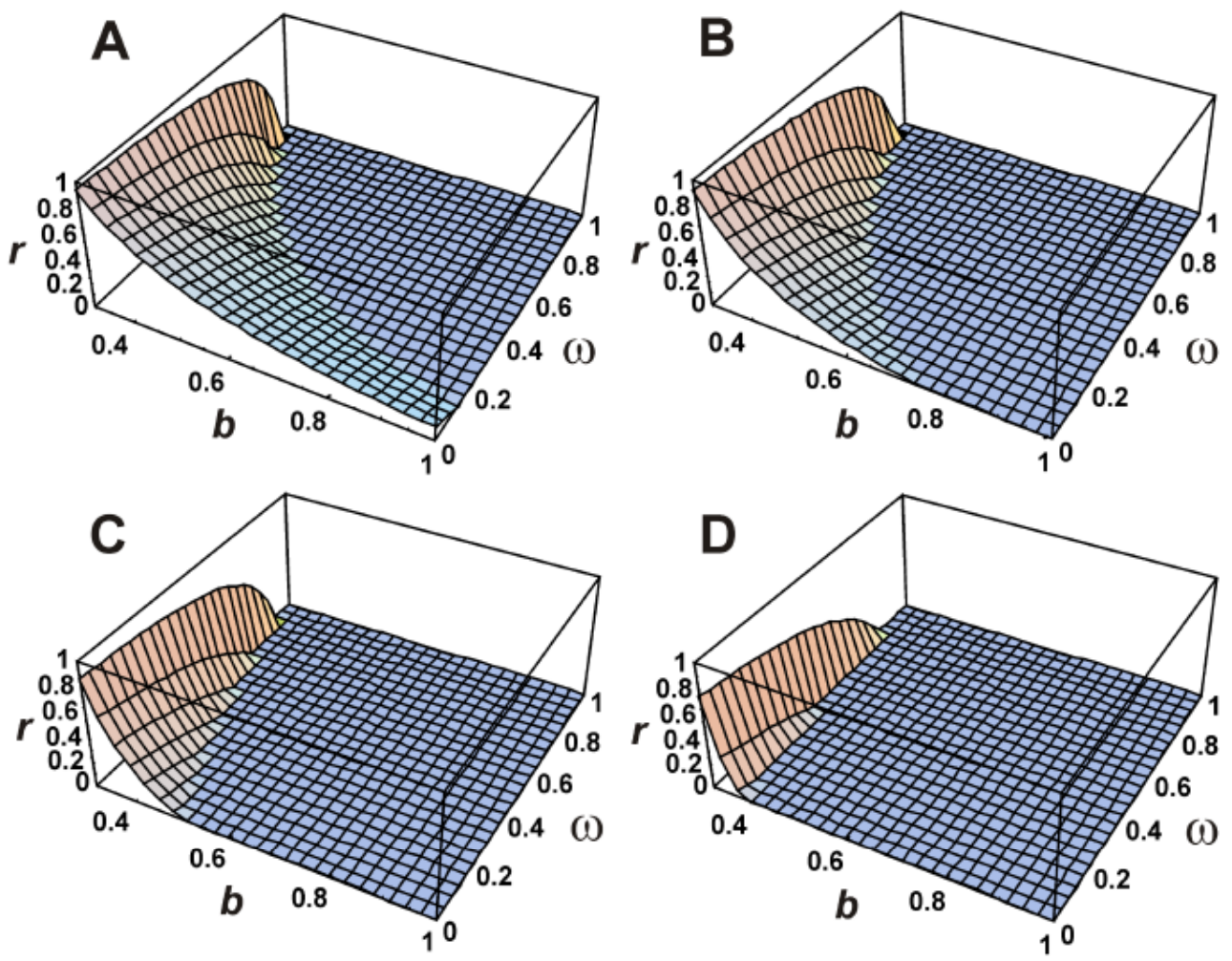

Fig. (3). Effect of probability of meeting the same person again $(\omega)$ and benefit of cooperation $(b)$ on the threshold relatedness $(r)$ required for maintaining cooperation for different values of probability of knowing other persons strategy $(q)$ when cooperators use both direct and indirect reciprocity. (A) $q=0.2,(\mathbf{B}) q=0.4,(\mathbf{C}) q=0.6$ and (D) $q=0.8$. Cost of cooperation $(c)$ is 0.3 .

kin selection can evolve cooperation from a population of defectors.

\section{Strong Reciprocity Among Kin}

Recent studies advocate that cooperation can be maintained if cooperators punish defectors. Because punishment itself is costly this prosocial form of reciprocity is called as strong reciprocity [8, 9]. In two person repeated PD interactions strong reciprocity can take the form of PunishBut-Don't-Tit (PBDT) strategy $[25,26]$. In a PBDT strategy, the player cooperates in the first move and from the second move cooperates with or without punishment depending on the opponent's strategy in the previous move. If the opponent cooperated in the previous move then PBDT plays cooperation without punishment. However, if the opponent has defected in the previous move then the PBDT plays cooperation with punishment. The cost of punishment is $x$ to the PBDT player and the punished defector plays $y$ (where $y$ $>x>0$ and $b-y<-c-x)$. Let $\omega$ be the probability that same two players will interact again. Two PBDT players will cooperate in all the interactions so,

$$
\begin{aligned}
E(P B D T \mid P B D T)= & (b-c)+\omega(b-c) \\
& +\omega^{2}(b-c)+\omega^{3}(b-c)+\ldots . .=\frac{b-c}{1-\omega}
\end{aligned}
$$

However, when in contest with the D player, PBDT player will cooperate without punishment in the first interaction but will cooperate with punishment in the subsequent interactions, so,

$$
\begin{aligned}
E(P B D T \mid D)=(-c) & +\omega(-c-x)+\omega^{2}(-c-x) \\
& +\omega^{3}(-c-x)+\ldots . .=-c+\frac{\omega(-c-x)}{1-\omega}
\end{aligned}
$$

While, the defector will get,

$$
\begin{aligned}
E(D \mid P B D T)=(b)+\omega & (b-y)+\omega^{2}(b-y) \\
& +\omega^{3}(b-y)+\ldots . .=b+\frac{\omega(b-y)}{1-\omega}
\end{aligned}
$$

Thus the payoff matrix can be given as,

\begin{tabular}{c|cc} 
& $P B D T$ & $D$ \\
\hline$P B D T$ & $\frac{(b-c)}{1-\omega}$ & $-c+\frac{\omega(-c-x)}{1-\omega}$ \\
$D$ & $b+\frac{\omega(b-y)}{1-\omega}$ & 0
\end{tabular}

PBDT will be stable against defectors when $\mathrm{E}$ $(\mathrm{PBDT} \mid \mathrm{PBDT})>\mathrm{E}(\mathrm{D} \mid \mathrm{PBDT})$, i.e. when $\omega>c / y$. However, PBDT players cannot invade a population of defectors as $\mathrm{E}(\mathrm{D} \mid \mathrm{D})>\mathrm{E}(\mathrm{PBDT} \mid \mathrm{D})$ for any $c>0$ and $x>0$ (except that members in different local groups who belong to the same clan have a potential resource of kin allies who change the expected pay-off and thus deter defectors).

Now consider that the interactions are among individuals with some degree of kinship, for then the penalty of the 
punishment and cost of punishment will also be shared by both the players proportional to degree of relatedness. Thus,

$$
\begin{gathered}
E(P B D T \mid P B D T)=(b-c)(1+r)+\omega(b-c)(1+r) \\
+\omega^{2}(b-c)(1+r)+\ldots . .=\frac{(b-c)(1+r)}{1-\omega} \\
E(P B D T \mid D)=(r b-c)+\omega(r b-c-r y-x) \\
+\ldots . .=(r b-c)+\frac{\omega(r b-c-r y-x)}{1-\omega} \\
E(D \mid P B D T)=(b-r c)+\omega(b-r c-y-r x) \\
+\ldots . .=(b-r c)+\frac{\omega(b-r c-y-r x)}{1-\omega}
\end{gathered}
$$

Thus the payoff matrix takes the form (Eq. 12).

PBDT will be stable against defectors when E $(\mathrm{PBDT} \mid \mathrm{PBDT})>\mathrm{E}(\mathrm{D} \mid \mathrm{PBDT})$, i.e. when $r>\frac{c-\omega y}{b+\omega x}$. Thus, punishment can reduce the threshold relatedness even when punishment is costly to the punisher so long as $y>0$ and $\omega>$ 0 .

Fig. (4) shows the threshold value of relatedness for different values of $\omega$ and $b$. PBDT can maintain cooperation when both benefit of cooperation and probability of meeting the same opponent is small. PBDT is far better than TFT in maintaining cooperation when benefit of cooperation is small (Figs. 2 and 4). However, PBDT can invade the population of defectors only if $r>\frac{c+\omega x}{b-\omega y}$. This condition implies that punishment is not a good mechanism to evolve cooperation as the relatedness required will be even more than kin selection working alone for any $y>x>0$. Thus, we cannot rely on strong reciprocity to evolve cooperation among defectors.

\section{Strong and Indirect Reciprocity Among Kin}

Similar to direct reciprocity, a major drawback of strong reciprocity by PBDT strategy is that it always cooperates without punishment with the opponent in the first round and will suffer costs unless there are group selected norms like those just described. In such a condition, knowing the strategy of the opponent by discrimination can always be a benefit as the PBDT strategy can punish the known defector even in the first round. If a player uses strategy involving both strong and indirect reciprocity (SRIR) then the payoff matrix can be derived as follows.

$$
\begin{aligned}
E(\text { SRIR } \mid \text { SRIR })= & (b-c)(1+r)+\omega(b-c)(1+r) \\
& +\omega^{2}(b-c)(1+r)+\ldots . .=\frac{(b-c)(1+r)}{1-\omega} \\
E(S R I R \mid D)= & {[(1-q)(r b-c)+q(r b-c-r y-x)] } \\
& +\omega(r b-c-r y-x)+\ldots . . \\
= & (b-r c-q y-q r x)+\frac{\omega(b-r c-y-r x)}{1-\omega} \\
E(D \mid S R I R)= & (1-q)(b-r c)+q(b-r c-y-r x)] \\
& +\omega(b-r c-y-r x)+\ldots . . \\
= & (r b-c-q r y-q x)+\frac{\omega(r b-c-r y-x)}{1-\omega}
\end{aligned}
$$

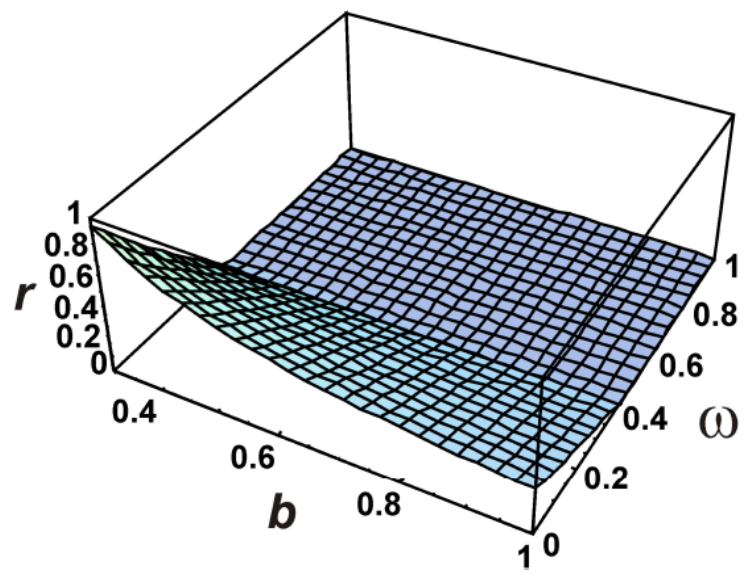

Fig. (4). Effect of probability of meeting the same person again $(\omega)$ and benefit of cooperation $(b)$ on the threshold relatedness $(r)$ required for maintaining cooperation when cooperators use PBDT strategy. Cost of cooperation $(c)$ is 0.3 , cost of punishing $(x)$ is 0.1 and penalty of punishment $(y)$ is 0.7 .

\begin{tabular}{c|cc} 
& $P B D T$ & $D$ \\
\hline \multirow{2}{*}{$P B D T$} & $\frac{(b-c)(1+r)}{1-\omega}$ & $(r b-c)+\frac{\omega(r b-c-r y-x)}{1-\omega}$ \\
$D$ & $(b-r c)+\frac{\omega(b-r c-y-r x)}{1-\omega}$ & 0
\end{tabular}




\begin{tabular}{c|cc} 
& $S R I R$ & $D$ \\
\hline SRIR & $\frac{(b-c)(1+r)}{1-\omega}$ & $(r b-c-q r y-q x)+\frac{\omega(r b-c-r y-x)}{1-\omega}$ \\
$D$ & $(b-r c-q y-q r x)+\frac{\omega(b-r c-y-r x)}{1-\omega}$ & 0
\end{tabular}

In the matrix form (Eq. 13).

SRIR will be stable against defectors when E $(\mathrm{SRIR} \mid \mathrm{SRIR}) \quad>\quad \mathrm{E} \quad(\mathrm{D} \mid \mathrm{SRIR}), \quad$ i.e. when $r>\frac{c-\omega y-q y(1-\omega)}{b+\omega x+q x(1-\omega)}$ Thus, SRIR can be a very effective strategy to main cooperation even when punishment is costly so long as players can discriminate between cooperators and defectors, play repeated interactions and penalty of punishment $y>0$.

Effect of $\omega, q$ and $b$ on the threshold value of $r$ required to evolve cooperation using SRIR strategy is shown in Fig. (5). Strong and indirect reciprocity among kin can be a very robust means, better than DRIR strategy, of maintaining cooperation when the benefit of cooperation is small. However, SRIR will invade the population of defectors only if $r>\frac{c+\omega x+q x(1-\omega)}{b-\omega y-q y(1-\omega)}$ which is worse than any other strategy.
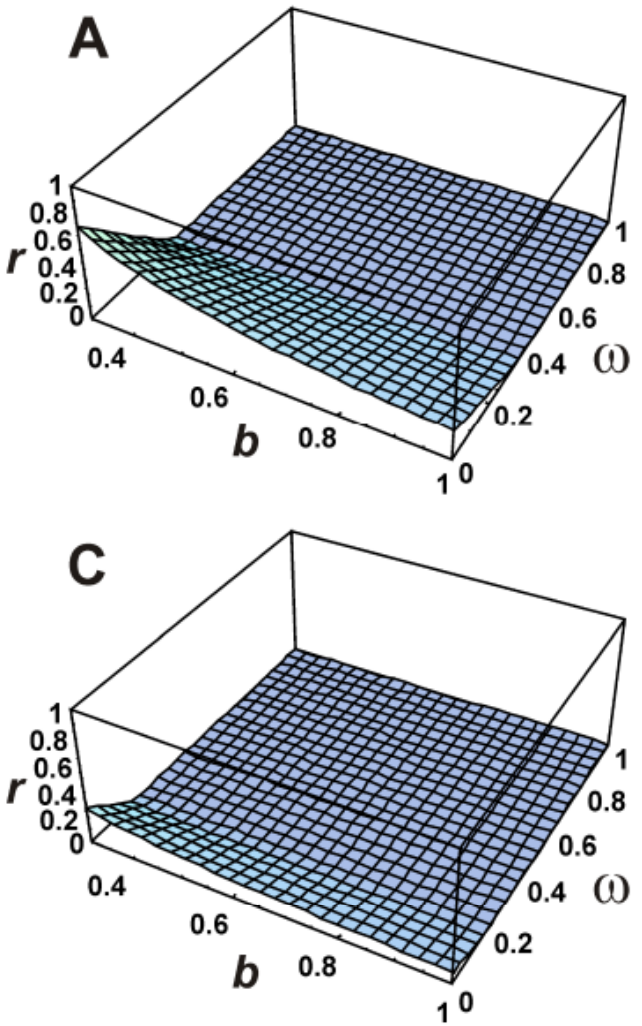

Fig. (5). Effect of probability of meeting the same person again $(\omega)$ and benefit of cooperation $(b)$ on the threshold relatedness $(r)$ required for maintaining cooperation for different values of probability of knowing other persons strategy $(q)$ when cooperators use both strong and indirect reciprocity. (A) $q=0.1,(\mathbf{B}) q=0.2,(\mathbf{C}) q=0.3$ and $(\mathbf{D}) q=0.4$. Cost of cooperation $(c)$ is 0.3 , cost of punishing $(x)$ is 0.1 and penalty of punishment $(y)$ is 0.7 . 
Table 2. Evolution, Maintenance and Invasion Criteria for Cooperation in Prisoner's Dilemma Played Between Kin Where ' $r$ ' is the Positive Assortment Probability

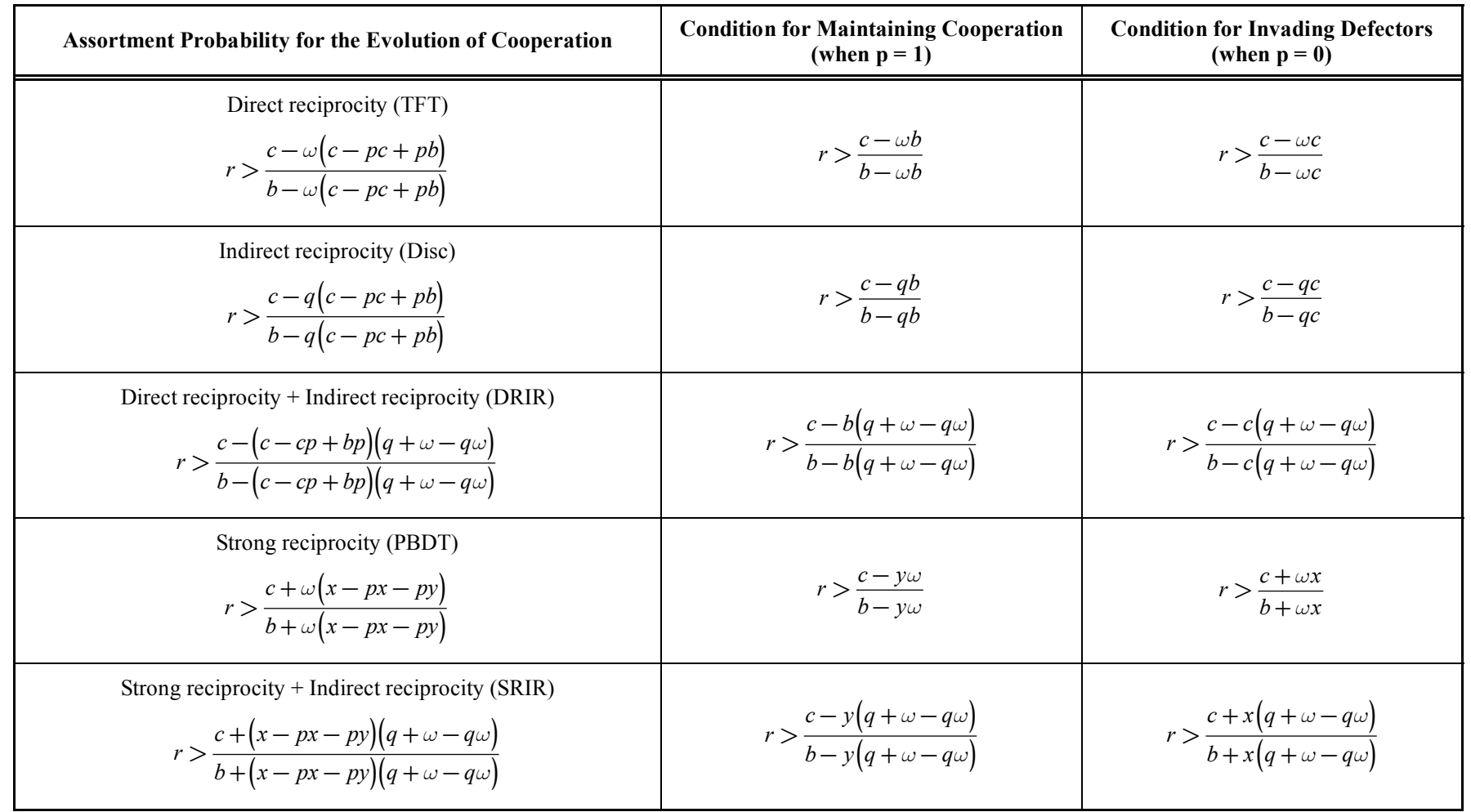

ment probability yields the same result. However, the conditions for evolution of cooperation and its maintenance with reciprocity among kin are different (Table 2). Comparison of the conditions for maintenance of cooperation and invasion of defectors for the two models reveal two interesting differences. First, in the case of strategies that rely on future interactions with the same opponent (TFT, DRIR, PBDT and SRIR) the condition for the invasion of defectors are dependent on the probability of the same two players meeting again $(\omega)$ in the case of positive assortment model, however, TFT and DRIR are independent of $\omega$ in the case of relatedness coefficient model. Second, $r$ required for the invasion of defectors in the positive assortment model is smaller than the relatedness coefficient model in the case of direct reciprocity (TFT), indirect reciprocity (Disc) and DRIR strategies.

The positive assortment model, however, suffers from one important problem. We will illustrate the problem by considering direct reciprocity among kin. A game between the TFT and D players will take the form of matrix (6). In the conventional approach to this game there are two pure strategy Nash Equilibriums, one at $(\mathrm{D}, \mathrm{D})$ and second at (TFT,TFT). Both these equilibriums are stable and one of them will be achieved in the population based on the initial frequency of the strategies. There is also a mixed strategy Nash Equilibrium which is unstable. A population of TFT players is stable against invasion by defectors. However, in a population of defectors, TFT cannot invade since $\mathrm{E}(\mathrm{D} \mid \mathrm{D})>$ $\mathrm{E}(\mathrm{TFT} \mid \mathrm{D})$, as $0>-c$ for any value of probability of interaction with the same opponent $(\omega)$. Thus, in the words of Axelrod and Hamilton [6], " $D$ is evolutionary stable no matter what is the probability of interaction continuing".

We will now consider direct reciprocity among kin. The invasion and stability criteria for direct reciprocity among kin by relatedness coefficient model and positive assortment model are given in Table $\mathbf{1}$ and Table $\mathbf{2}$ respectively. The plots of these criteria (Fig. 6) suggest that the stability criteria do not differ qualitatively except for the fact that the relationship is non-linear in the case of positive assortment model while it is linear in the case of relatedness coefficient model. However there is a difference in the invasion criteria. The positive assortment model makes a prediction that if the probability of meeting the same person is unity (i.e. if the same two players interact infinitely) TFT can invade a population of $\mathrm{D}$ players even when positive assortment $r$ is zero (Fig. 6B). We have already seen that this is not possible because $\mathrm{D}$ is evolutionary stable whatever the chances of interaction continuing. On the contrary, in the relatedness coefficient model invasion criteria is independent of the probability of meeting the same opponent again (Fig. 6A). This is intuitively clear since TFT as a sole invader will always meet defectors and will fail to invade at any value of $\omega$. It is therefore not at all surprising that $\omega$ does not appear in the invasion criteria when a sole TFT is invading a population of defectors. However, as soon as there is a sizable population of TFT, $\omega$ becomes an important determinant. Thus, relatedness coefficient model suggests that initial invasion of D by TFT players can only rely on the relatedness coefficient and TFT can invade only when $r>c / b$. This is compatible with the assertion by Axelrod and Hamilton [6, pp. 1394]. 

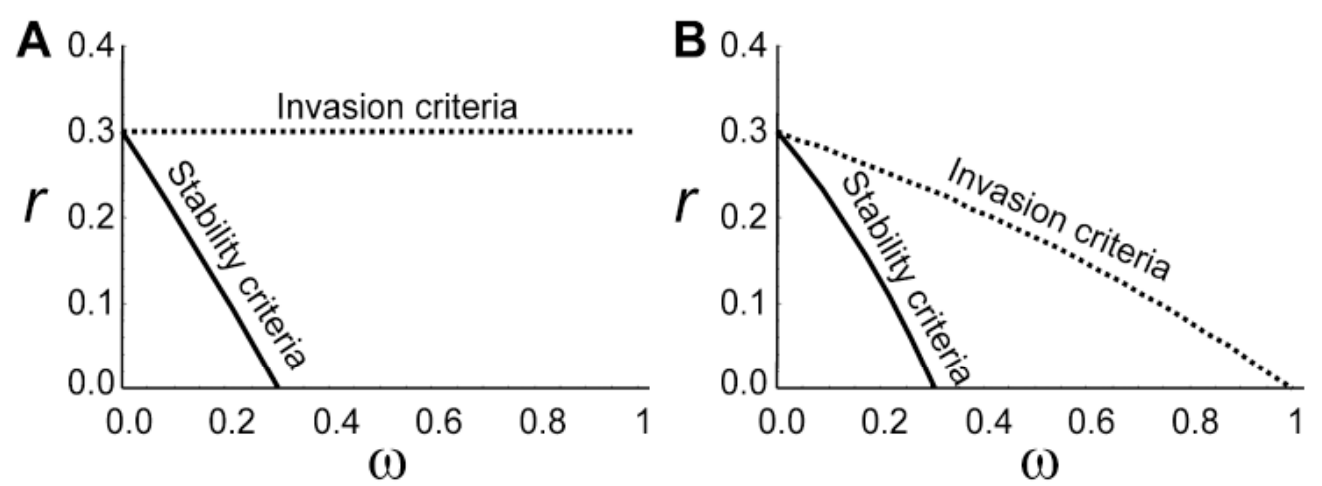

Fig. (6). Stability and invasion criteria for cooperation maintained by direct reciprocity among kin. (A) Based on the relatedness coefficient model (Table 1) and (B) based on positive assortment model (Table 2). Other parameters are $b=1$ and $c=0.3$.

\section{DISCUSSION}

In the light of the recent conflict over priorities and favoring one or few mechanisms of evolution of cooperation over others [11-15], our findings depict that a synthesis, where different mechanisms of evolution of cooperation coexist is far more robust to defection than any mechanism working alone. We have shown with simple game theoretical models that when group selection operates in between-group competition and kin selection works to resolve within-group conflicts, the resultant model is superior in evolving cooperation and maintaining it than any one strategy working alone. Furthermore, we showed that initially within group conflicts can be overcome only by kin selection and not reciprocity. However, once common, different types of reciprocities can maintain cooperation at high levels even when average relatedness among individuals is small. Our analysis suggests that when different mechanisms of evolution of cooperation work together, cooperation can be maintained in the population even when the average relatedness is low, groups are large and the benefits of cooperation are low.

Two approaches are suggested for studying kin selection namely relatedness coefficient model and positive assortment model. We tested both the models. Our analysis suggests that the stability criteria for different types of reciprocities do not differ much in both the models, however, the invasion criteria differ significantly. Nonetheless, taking direct reciprocity, using TFT strategy, as an example we showed that positive assortment model makes an impossible prediction about invasion of defector population. Positive assortment model suggests that TFT can invade a population of defectors, even when positive assortment probability is zero, given that the probability of meeting the same opponent is unity (Fig. 6B). This is not possible because defection is evolutionary stable irrespective of the probability of meeting the same opponent again [6]. On the contrary, relatedness coefficient model suggests that TFT can invade the population of defectors only through kin selection when $r>c / b$ (Fig. 6A). Our analysis is not only in concurrence with the suggestions given by Axelrod and Hamilton [6] it also gives a mathematical basis for their argument. Our analysis also suggests that at least in the matrix games, the positive assortment approach should be used with caution.

There are two paths suggested for the evolution of social and eusocial systems. In the subsocial route societies originate from familial units initially composed of parents and offspring, while in the semisocial route societies originate through associations of individuals, related or unrelated, from the same generation [28]. Even though empirical evidence of kin selection in diverse group of organisms is overwhelming [29-35], many researchers have renounced subsocial route based on the fact that in a variety of societies the average relatedness is lower than expected [12, 36]. Furthermore, it has been shown that many social organizations also rely heavily on reciprocity [37, 38]. Anderson [39] has argued that "high genetic relatedness does not mean that kin selection is crucial for the evolution of eusocial systems rather reciprocity might have played a vial role, while high genetic relatedness can be just an outcome of evolution of eusociality". On the contrary, our analysis strongly supports the subsocial route for the evolution of social systems.

We propose that social and eusocial systems initially probably evolved among closely related individuals. This is because our analysis shows that none of the proposed mechanisms of reciprocity can evolve cooperation in unrelated egoistic individuals unless reinforced by memory of previous interactions, discrimination and coercion. Additional direct evidence to our proposal comes from a recent study by Hughes et al. [40] who showed that monogamy is prevalent in the ancestral eusocial systems. Thus, the initial social systems were made up of subsocial groups consisting of close relatives like parents and offspring. At this stage both kin selection at the individual level and group selection at the group level might have played a pivotal role to give rise to social groups as we have shown that kin selection and group selection together can work far better than either of the strategies alone. Such subsocial groups might have been regularly threatened by intergroup conflicts, predation and parasitism. While it was possible to succeed in intergroup conflicts and reduce predation merely by increased group size, threat of parasitism necessitated the selection for genetic heterogeneity. We suspect that intergroup conflict, threat due to predation or parasitism, and generation of collective common goods were responsible for the increase in the group size of social systems.

Increase in group size might have occurred in two different ways. In the case of organisms with high reproductive rates, reproductive rate alone is sufficient to increase the group size. However, in the case of organisms with low reproductive rates, group merging might have played an important role. Interestingly such group merging for increa- 


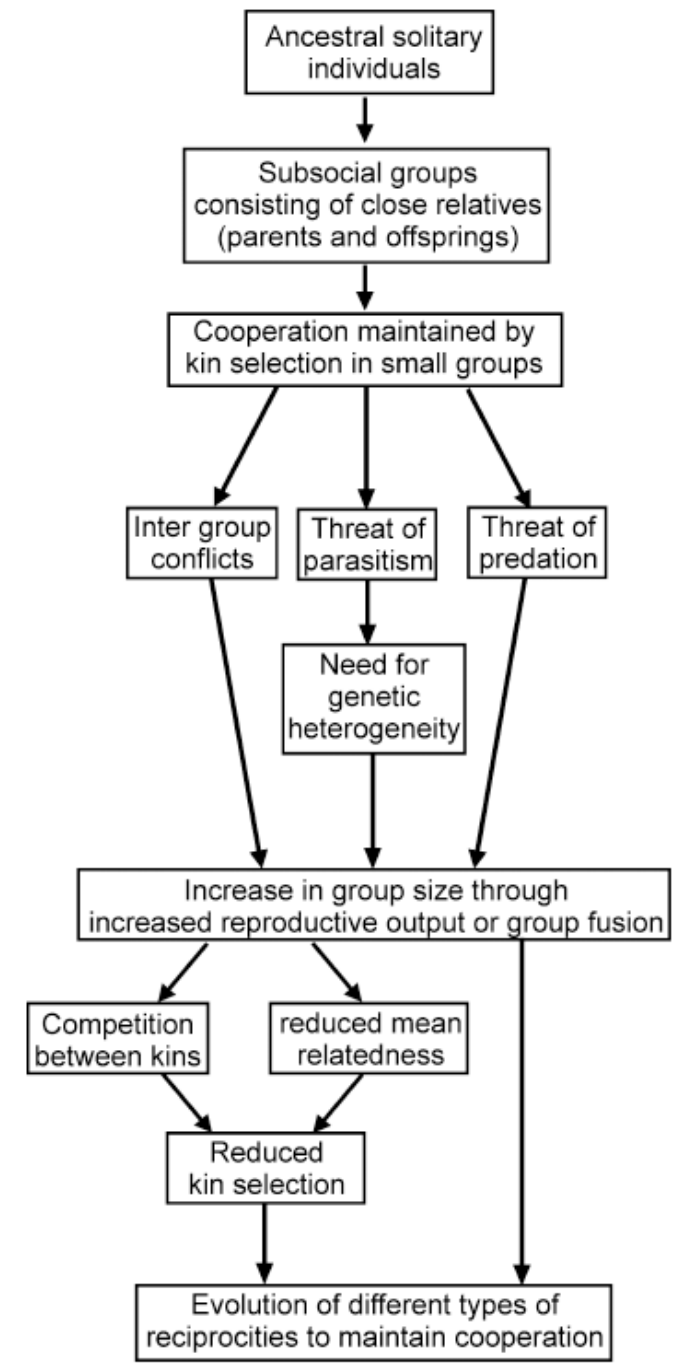

Fig. (7). Possible route to the evolution of social and eusocial systems.

sing the reproductive benefit is reported in social amoebae [41]. Group merging, however, would inevitably decrease the genetic relatedness among individuals. Decreased genetic relatedness and low individual benefits due to large group sizes might have led to the evolution of reciprocity, which is more efficient in maintaining cooperation in large groups.

In the case of organisms with high reproductive rates, two problems might have occurred. First, when genetic relatedness was high, competition between kin might have contributed to conflicts [42] which led to the evolution of different types of reciprocities [43]. Secondly, as group sizes started expanding, groups were forced to maintain genetic heterogeneity, owing to the threats of parasitism. At this stage kin selection would have been insufficient to maintain cooperation and different forms of reciprocities evolved to cope up with the scenario. Our proposed route for the evolution of social and eusocial systems is given in Fig. (7).

Once cooperation is common different forms of reciprocities can maintain cooperation even when genetic relatedness is low. However, inability of reciprocity to invade defectors makes reciprocity an unlikely candidate for the early evolution of social systems. We therefore believe that social organizations evolved through subsocial route among genetically related individuals in small groups and different types of reciprocities evolved later to maintain cooperation when relatedness was lowered through group expansion and genetic heterogeneity. This process is especially effective when cooperation leads to changes in ecology that effectively increase resources (as is illustrated among humans by the development of agriculture).

Various empirical findings support our view. Hughes et al. [40] showed that ancesters of eusocial wasps consisted of subsocial groups with close relatives like parents and offspring and kin selection could explain cooperation among the individuals. However, as pointed out by Ratnieks and Wenseleers [44] intermediate levels of relatedness found within modern-day insect societies are too low to directly cause the extreme levels of cooperation among individuals. Instead, their cooperation seems to be enforced by social sanctions in terms of punishment and policing by individuals $[44,45]$. Thus with expansion of groups and increased genetic heterogeneity led to the increase in the coercion in insects and other vertebrates [44].

Another empirical evidence of collective punishment of a defector within group with close and distantly related kin comes from social system of Antarctic Penguins. The film 
"March of the Penguins" (Warner Brothers Entertainment Inc., 2005) provided a visual record of Penguin social behavior in the species' native habitat of Antarctica. Penguin females give birth to each year's fledglings at the same time of year. All females in a local group huddle together-each with her fledgling in a pouch over their feet-to provide protection from the howling winds and cold temperature. If a fledgling falls out of its mother's pouch and froze to death, after inspecting her dead fledgling, the mother approaches the huddled group of other Penguin mothers and attempt to steal a fledgling from its mother's pouch. All of the females in the group immediately attacked the "defector" from the species-typical rules, beating her for the act of stealing the baby. In short, this film provides empirical evidence of a collective action to defend a collective interest.

Human social system, which has reached the pinnacle of cooperative behavior, also supports the predictions of our model. Firstly, it is now clear that humans have evolved mechanisms to detect kin [34, 46, 47]. Rushton [46, 47] has argued that humans have a tendency to recognize genetic similarity to give preferential treatment to kin. This behavior gives a biological substrate for ethnocentrism, enabling group selection to occur. Our journey to the modern nuclear families can be traced back to the hunter gatherers living in small clans and tribes. The marriage systems in several clans and tribes were influenced by kin networks $[48,49]$. It is therefore possible that subsocial route had a complete or atleast a partial role in the primitive sociality. Social structures and leadership within the local residential group might have further reinforced the subsocial route. As ethologists and anthropologists have found, among other mammals whose groups are led by an alpha male, cooperative behavior among humans and great apes was greatly influenced by leader who was bonded with and supported by other males (some of whom were kin and whose support increases the leader's reproductive success). As an example, Yanomamo clan in Venezuela, who typically had multiple wives to insure that every lineage in the clan had some offspring bearing the headman's genes [49]. The leaders of many preliterate tribes (such as many Native American chieftains) had similarly greater than average mating opportunities, following customs that both enhanced the chief's power to maintain control over the clan or tribe and integrated the tribe's competing lineages.

Note, however, that the evolution of human language and naming system-by permitting vastly increased precision in the recognition of distant kin-served as a collective good, permitting humans to hunt larger game and succeed in between-species competition as well as developing technologies allowing survival in otherwise marginal environments. Language (a collective good) creates within-group solidarity that extends the kin model, creating the potential of betweengroup alliance against outside groups (e.g., tribal warfare uniting otherwise competing bands within each preliterate tribe). Such between-group alliance decreases the benefits of kin selection but increase the benefits of cooperation if groups adhere to certain laws. Laws to sustain cooperation either by tit-for-tat or severe punishment can be traced to ancient human civilizations. A good example can be found in chapter 19 of the book of Deuteronomy in the Old Testament governing revenge for murder among the Hebrews. This example illustrates the robust benefits of linking kinship with group selection if it takes the form of socially accepted rules and norms as well as leadership capable of organizing group punishment of defectors. Another important contribution of language was means to gossip. In fact the need to gossip has been argued to be one of the major selective forces for the evolution of language [50]. Gossip makes it possible to gain information about the behaviour of others without actual eavesdropping. Thus, indirect reciprocity, or reputation based reciprocity, works better in the presence of language than its absence. Indeed, only such processes can explain the very large increase in the size of human societies from the hunter-gatherer band that characterized thousands of years of human evolution and social development leading to the modern nation-state (which is a product of the last three centuries and hence but a moment in the overall sweep of human evolution).

\section{ACKNOWLEDGEMENT}

We are thankful to two anonymous referees and Kishore Pawar for valuable suggestions on the earlier draft of the manuscript.

\section{REFERENCES}

[1] Wilson DS. A theory of group selection. Proc Natl Acad Sci USA 1975; 72: 143-6.

[2] Dawkin R. The selfish gene. Oxford, UK: Oxford University Press 1976

[3] Maynard SJ. The origin of altruism. Nature 1998; 393: 639-40.

[4] Hamilton WD. The genetic evolution of social behavior I and II. J Theor Biol 1964; 7: 1-52.

[5] Trivers RL. The evolution of reciprocal altruism. Q Rev Biol 1971; 46: 35-57.

[6] Axelrod R, Hamilton WD. The evolution of cooperation. Science 1981; 211: 1390-6.

[7] Nowak MA, Sigmund K. Evolution of indirect reciprocity by image scoring. Nature 1998; 393: 573-7.

[8] Gintis H. Strong reciprocity and human sociality. J Theor Biol 2000; 206: 169-79.

[9] Fehr E, Gachter S. Altruistic punishment in humans. Nature 2002; 415: 137-40.

[10] Griffin AS, West SA. Kin selection: fact and fiction. Trends Ecol Evol 2002; 17: 15- 21.

[11] Wilson EO. Kin selection as the key to altruism: its rise and fall. Soc Res 2005; 72: 159-68.

[12] Wilson EO, Hölldobler B. Eusociality: origin and consequences. Proc Natl Acad Sci USA 2005; 102: 13367-71.

[13] Foster KR, Wenseleers T, Ratnieks FLW. Kin selection is the key to altruism. Trends Ecol Evol 2006; 21: 57-60.

[14] Fletcher JA, Zwick M, Doebeli M, Wilson DS. What's wrong with inclusive fitness? Trends Ecol Evol 2006; 21: 597-8.

[15] Foster KR, Wenseleers T, Ratnieks FLW, Queller DC. There is nothing wrong with inclusive fitness. Trends Ecol Evol 2006; 21: 599-600.

[16] Lehmann L, Keller L, West S, Roze D. Group selection and kin selection: two concepts but one process. Proc Natl Acad Sci USA 2007; 104: 6736-9.

[17] Lehmann L, Keller L. The evolution of cooperation and altruism-a general framework and a classification of models. J Evol Biol 2006; 19: 1365-76.

[18] Marshall JAR, Rowe JE. Kin selection may inhibit the evolution of reciprocation. J Theor Biol 2003; 222: 331-5.

[19] Fletcher JA, Zwick M. Unifying the theories of inclusive fitness and reciprocal altruism. Am Nat 2006; 168: 252-62.

[20] Gruter M, Masters RD. Ostracism as a social and biological phenomenon: an introduction. Ethol Sociobiol 1986; 7: 149-58.

[21] Grafen A. The hawk-dove game played between relatives. Anim Behav 1979; 27: 905-7. 
[22] Milchtaich I. Comparative statics of games between relatives. Theor Popul Biol 2006; 69: 203-10.

[23] Nowak MA. Five rules for the evolution of cooperation. Science 2006; 314: 1560-3.

[24] Maynard Smith J. The theory of games and the evolution of animal conflicts. J Theor Biol 1974; 47: 209-21.

[25] Dahanukar N, Ganguly B. Punish but don't tit: an evolutionary stable strategy in iterated Prisoners' Dilemma. Logic Biol 2004; 4: 60-7.

[26] Dahanukar N. Cooperation in Prisoner's Dilemma: direct versus strong reciprocity. Logic Biol 2005; 5: 188-99.

[27] McElreath R, Boyd R. Mathematical models of social evolution: a guide for the perplexed. University of Chicago Press; 2007.

[28] Throne BL. Evolution of eusociality in termites. Ann Rev Ecol Syst 1997; 28: 27-54.

[29] Mehdiabadi NJ, Jack CN, Farnham TT, et al. Kin preference in a social microbe. Nature 2006; 442: 881-2.

[30] Foster KR, Ratnieks FL. Paternity, reproduction and conflict in vespine wasps: a model system for testing kin selection predictions. Behav Ecol Sociobiol 2001; 50: 1-8.

[31] Khan MZ, Walters JR. An analysis of reciprocal exchange of helping behavior in the red-cockaded woodpecker (Picoides borealis). Behav Ecol Sociobiol 2000; 47: 376-81.

[32] Krakauer AH. Kin selection and cooperative courtship in wild turkeys. Nature 2005; 434: 69-72.

[33] Silk JB. Kin selection in primate groups. Int J Primatol 2002; 23 : 849-75.

[34] Lieberman D, Tooby J, Cosmides L. The architecture of human kin detection. Nature 2007; 445: 727-31.

[35] Dudley SA, File AL. Kin recognition in an annual plant. Biol Lett 2007; 3: 435-8.

[36] Gadagkar R. The haplodiploidy threshold and social evolution. Curr Sci 1990; 59: 374-6.

[37] Wenseleers T, Badcock NS, Erven K, et al. A test of worker policing theory in an advanced eusocial wasp, Vespula rufa. Evolution 2005; 59: 1306-14
[38] Wenseleers T, Helantera H, Hart A, Ratnieks FLW. Worker reproduction and policing in insect societies: an ESS analysis. J Evol Biol 2004; 17: 1035-47.

[39] Anderson M. The evolution of eusociality. Ann Rev Ecol Syst 1984; $15: 165-89$.

[40] Hughes WOH, Oldroyd BP, Beekman M, Ratnieks FLW. Ancestral monogamy shows kin selection is key to the evolution of eusociality Science 2008; 320: 1213-6

[41] Fortunato A, Strassmann JE, Santorelli L, Queller DC. Cooccurrence in nature of different clones of the social amoeba, Dictyostelium discoideum. Mol Ecol 2003; 12: 1031-8.

[42] West SA, Murray MG, Machado CA, Griffin AS, Herre EA. Testing Hamilton's rule with competition between relatives. Nature 2001; 409: 510-3.

[43] Ratnieks FLW, Foster KR, Wenseleers T. Conflict resolution in insect societies. Ann Rev Entomol 2006; 51: 581-608.

[44] Ratnieks FLW, Wenseleers T. Altruism in insect societies and beyond: voluntary or enforced? Trends Ecol Evol 2008; 23: 45-52.

[45] Wenseleers T, Ratnieks FLW. Enforced altruism in insect societies. Nature 2006; 444: 50.

[46] Rushton JP. Genetic similarity, human altruism, and group selection. Behav Brain Sci 1989; 12: 503-59.

[47] Rushton JP. Inclusive fitness in human relationships. Biol J Linn Soc 2009; 96: 8-12.

[48] Bengtsson T, Mineau GP (Ed.) Kinship and demographic behavior in the past. USA: Springer; 2008.

[49] Fielder C, King C. Sexual Paradox: Complementarity, Reproductive Conflict and Human Emergence. Courtauld Institute, London; 2009. Online reference: http://www.dhushara.com/paradoxhtm/warrior. htm [Accessed: 30 May 2009].

[50] Dunbar R. Theory of mind and the evolution of language. In: Hurford JR, Studdert-Kennedy M, Knight C, Eds, Approaches to the Evolution of Language: Social and Cognitive Bases. Cambridge: Cambridge University Press 1998; pp. 92-110.

(C) Dahanukar and Watve; Licensee Bentham Open.

This is an open access article licensed under the terms of the Creative Commons Attribution Non-Commercial License (http://creativecommons.org/licenses/by$\mathrm{nc} / 3.0 /$ ), which permits unrestricted, non-commercial use, distribution and reproduction in any medium, provided the work is properly cited. 